Supplement of Hydrol. Earth Syst. Sci., 20, 3183-3191, 2016

http://www.hydrol-earth-syst-sci.net/20/3183/2016/

doi:10.5194/hess-20-3183-2016-supplement

(C) Author(s) 2016. CC Attribution 3.0 License.

(c) (i)

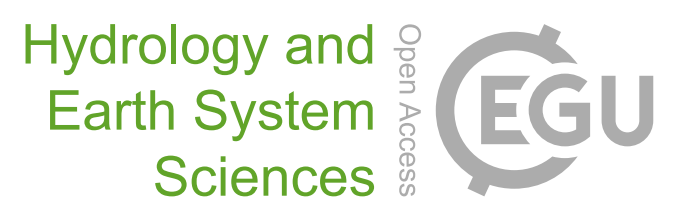

Supplement of

\title{
Technical note: Multiple wavelet coherence for untangling scale-specific and localized multivariate relationships in geosciences
}

Wei Hu and Bing Cheng Si

Correspondence to: Wei Hu (wei.hu@ plantandfood.co.nz) and Bing Cheng Si (bing.si@usask.ca)

The copyright of individual parts of the supplement might differ from the CC-BY 3.0 licence. 


\section{S1 Calculation of smoothed auto- and cross-wavelet power spectra}

In this section, we will only introduce the basics related to the calculation of smoothed

19 auto- and cross-wavelet power spectra. Detailed information on the calculations of

20 wavelet coefficients, cross-wavelet power spectra, and bivariate wavelet coherence can

21 be found elsewhere (Kumar and Foufoula-Georgiou, 1997; Torrence and Compo, 1998;

22 Torrence and Webster, 1999; Grinsted et al., 2004; Das and Mohanty, 2008; Si, 2008).

23 The smoothed auto- and cross-wavelet power spectra require the calculation of wavelet

24 coefficients, at different scales and spatial (or temporal) locations, for the response

25 variable and all predictor variables. For convenience, only spatial variables will be

26 referred to, as temporal variables can be similarly analyzed.

27 The continuous wavelet transform (CWT) of a spatial variable $X_{1}$ of length $N\left(X_{1 h}\right.$,

$28 h=1,2, \ldots, N)$ with equal incremental distance $\delta x$, can be calculated as the convolution

29 of $X_{1 h}$ with the scaled and normalized wavelet (Torrence and Compo, 1998)

$$
W^{X_{1}}(s, \tau)=\sqrt{\frac{\delta x}{s}} \sum_{\tau=1}^{N} X_{1 h} \psi\left[(h-\tau) \frac{\delta x}{s}\right],
$$

31 where $W^{X_{1}}(s, \tau)$ is the wavelet coefficient of spatial variable $X_{1}$ at scale $s$ and location

$32 \tau$, and $\psi[]$ is the mother wavelet function. The Morlet wavelet is used in the CWT

33 because it allows for the identification of both location-specific amplitude and phase

34 information at different scales in a spatial series (Torrence and Compo, 1998). The

35 Morlet wavelet can be expressed as (Grinsted et al., 2004)

$$
\psi(\eta)=\pi^{-1 / 4} e^{i \omega \eta-0.5 \eta^{2}}
$$

37 where $\omega$ and $\eta$ are the dimensionless frequency and space $(\eta=s / x)$, respectively. 
38 The auto-wavelet power spectrum of spatial variable $X_{1}$ can be expressed as

$$
W^{X_{1}, X_{1}}(s, \tau)=W^{X_{1}}(s, \tau) \overline{W^{X_{1}}(s, \tau)},
$$

40 where $\overline{W^{X_{1}}(s, \tau)}$ is a complex conjugate of $W^{X_{1}}(s, \tau)$. Therefore, Eq. (3) can also be

41 expressed as the squared amplitude of $W^{X_{1}}(s, \tau)$, which is

$$
W^{X_{1}, X_{1}}(s, \tau)=\left|W^{X_{1}}(s, \tau)\right|^{2} .
$$

43 The cross-wavelet spectrum between spatial variables of $Y$ and $X_{1}$ can be defined as

$$
W^{Y, X_{1}}(s, \tau)=W^{Y}(s, \tau) \overline{W^{X_{1}}(s, \tau)},
$$

45 where $W^{Y}(s, \tau)$ is the wavelet coefficient of spatial variable $Y$.

46 Both the auto- and cross-wavelet spectra can be smoothed using the method suggested

47 by Torrence and Compo (1998),

$$
\overleftrightarrow{W}(s, \tau)=\mathrm{SM}_{\text {scale }}\left[\mathrm{SM}_{\text {space }}(W(s, \tau))\right] \text {, }
$$

49 where $\overleftrightarrow{(\cdot)}$ is a smoothing operator. $\mathrm{SM}_{\text {scale }}$ and $\mathrm{SM}_{\text {space }}$ indicate the smoothing along the

50 wavelet scale axis and spatial distance, respectively ( $\mathrm{Si}, 2008)$. The $\overleftrightarrow{W}$ is the normalized

51 real Morlet wavelet and has a similar footprint as the Morlet wavelet

$$
\frac{1}{s \sqrt{2 \pi}} e^{\left.\left(-\tau^{2}\right)\left(2 s^{2}\right)\right)} .
$$

53 Therefore, the smoothing along spatial distance can be calculated as

$$
\operatorname{SM}_{\text {scale }}(W(s, \tau))=\left.\sum_{k=1}^{N}\left(W(s, \tau) \frac{1}{s \sqrt{2 \pi}} e^{\left.\left(-(\tau-x)^{2}\right)^{2}\left(2 s^{2}\right)\right)}\right)\right|_{s} \text {, }
$$


55 where $\left.\right|_{s}$ represents a fixed $s$ value. The Fourier transform of Eq. (7) is $e^{\left(-2 s^{2} \omega^{2}\right)}$.

56 Therefore, Eq. (8) can be implemented using Fast Fourier Transform (FFT) and Inverse

57 Fast Fourier Transform (IFFT) based on the convolution theorem, and is written as

$58 \quad \operatorname{SM}_{\text {scale }}(W(s, x))=\operatorname{IFFT}\left(\operatorname{FFT}(W(s, x))\left(e^{\left(-2 s^{2} \omega^{2}\right)}\right)\right)$.

59 The smoothing along scales is then written as [Torrence and Compo, 1998]

$60 \mathrm{SM}_{\text {scale }}\left(W\left(s_{k}, x\right)\right)=\left.\frac{1}{2 m+1} \sum_{l=k-m}^{k+m}\left(\mathrm{SM}_{\text {space }}\left(W\left(s_{l}, x\right)\right) \Pi\left(0.6 s_{l}\right)\right)\right|_{x}$,

61 where $\Pi$ is the rectangle function, $\left.\right|_{x}$ indicates a fixed $x$ value, and $l$ is the index for the

62 scales. The coefficient of 0.6 is the empirically determined scale decorrelation length for

63 the Morlet wavelet (Torrence and Compo, 1998). 


\section{S2 Matlab code for MWC (mwc.m)}

$66 \%$ This is a Matlab code (mwc.m) for calculating multiple wavelet coherence.

$67 \%$ Please copy the following content into a txt file and rename it to "mwc.m" prior to running.

69 function varargout $=\mathrm{mwc}(\mathrm{X}, \mathrm{varargin})$

$70 \%$ Multiple Wavelet coherence

$71 \%$ Creates a figure of multiple wavelet coherence

$72 \%$ USAGE: [Rsq,period,scale,coi,sig95]=mwc(X,[,settings])

$73 \%$

74 \% Input: X: a matrix of multiple variables equally distributed in space

$75 \%$ or time. The first column corresponds to the dependent variable,

$76 \%$ and the second and consequent columns are independent variables.

$77 \%$

78 \% Settings: Pad: pad the time series with zeros?

$79 \%$ Dj: Octaves per scale (default: '1/12')

$80 \%$. S0: Minimum scale

$81 \%$ J1: Total number of scales

$82 \%$. Mother: Mother wavelet (default 'morlet')

$83 \%$. MaxScale: An easier way of specifying J1

$84 \%$. MakeFigure: Make a figure or simply return the output.

$85 \%$. BlackandWhite: Create black and white figures

$86 \%$ AR1: the ar1 coefficients of the series

$87 \%$. (default='auto' using a naive ar1 estimator. See arlnv.m)

$88 \%$. MonteCarloCount: Number of surrogate data sets in the significance calculation. (default=1000)

89

$90 \%$ Settings can also be specified using abbreviations. e.g. ms=MaxScale.

$91 \%$ For detailed help on some parameters type help wavelet.

$92 \%$ Example:

$93 \% \quad \mathrm{t}=[1: 200]$;

$94 \% \operatorname{mwc}\left(\left[\sin (\mathrm{t}), \sin \left(\mathrm{t} . * \cos \left(\mathrm{t}^{*} .01\right)\right), \cos \left(\mathrm{t} . * \sin \left(\mathrm{t}^{*} .01\right)\right)\right]\right)$

95

$96 \%$ Please acknowledge the use of this software package in any publications,

$97 \%$ by including text such as:

98

$99 \%$ "The software for the multiple wavelet coherence was provided by W. Hu

$100 \%$ and B. Si, and is available in the Supplement of $\mathrm{Hu}$ and $\mathrm{Si} \mathrm{(2016)."}$

$101 \%$ and cite the paper:

$102 \%$ "Hu, W., and B. Si (2016), Technical Note: Multiple wavelet coherence for untangling scale-specific and localized

$103 \%$ multivariate relationships in geosciences, Hydrol. Earth Syst. Sci., volume and page numbers to be allocated." 


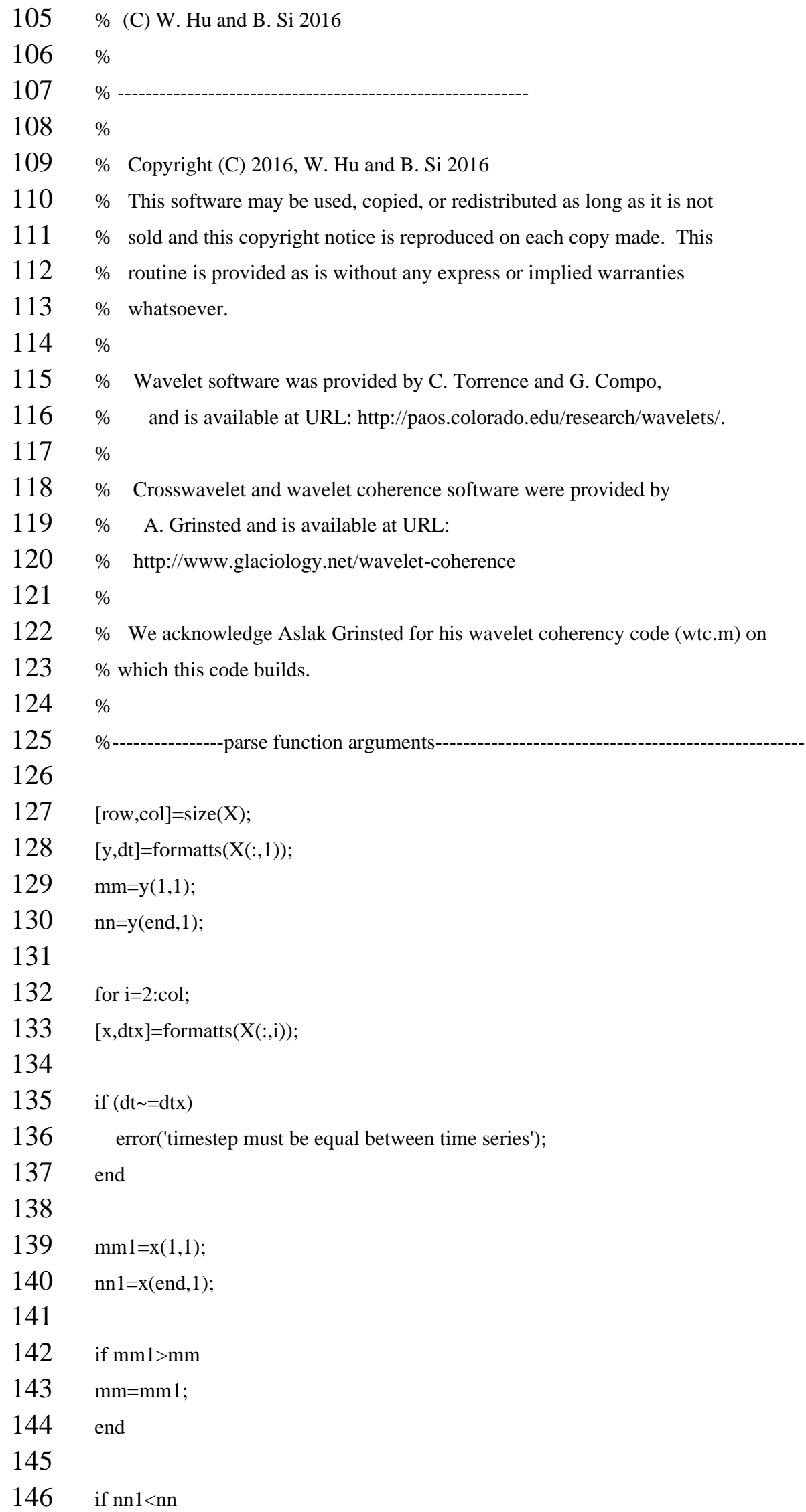


$\mathrm{nn}=\mathrm{nn} 1 ;$

148 end

149

150

$\mathrm{x} 1(:,(\mathrm{i}-1))=\mathrm{x}(:, 1)$;

$151 \quad \mathrm{x} 2(:,(\mathrm{i}-1))=\mathrm{x}(:, 2)$;

152

153 end

154

155

$\mathrm{t}=(\mathrm{mm}: \mathrm{dt}: \mathrm{nn})^{\prime}$

156

157

158

159

\%common time period

160

if length $(\mathrm{t})<4$

161

error('The three time series must overlap.');

162

163

end

164

165

$\mathrm{n}=$ length $(\mathrm{t})$;

166

\%----------default arguments for the wavelet transform-----------

167

Args=struct('Pad', $1, \ldots \quad \%$ pad the time series with zeroes (recommended)

168

'Dj', $1 / 12, \ldots \%$ this will do 12 sub-octaves per octave

169

'S0', $2 * \mathrm{dt}, \ldots \quad \%$ this says start at a scale of 2 years

170

171

172

173

174

175

176

177

178

'J1',[],...

'Mother','Morlet', ...

'MaxScale',[],... \%a more simple way to specify $\mathrm{J} 1$

'MakeFigure',(nargout==0),...

'MonteCarloCount',1000,...

'BlackandWhite', $0, \ldots$

179

180

'AR1','auto',,...

'ArrowDensity',[30 30],...

'ArrowSize', $1, \ldots$

'ArrowHeadSize',1);

181

182

183

184

185

186

Args=parseArgs(varargin,Args, $\{$ 'BlackandWhite' $\}$ );

187

if isempty(Args.J1)

if isempty(Args.MaxScale)

Args.MaxScale $=(n * .17) * 2 * d t ; \%$ auto maxscale;

end

188 end 
$\operatorname{ad}=$ mean(Args.ArrowDensity);

190 Args. ArrowSize=Args.ArrowSize*30*.03/ad;

$191 \%$ Args.ArrowHeadSize=Args.ArrowHeadSize*Args.ArrowSize*220;

192 Args.ArrowHeadSize=Args.ArrowHeadSize*120/ad;

193

194

if $\sim$ strcmpi(Args.Mother,'morlet')

195 warning('MWC:InappropriateSmoothingOperator','Smoothing operator is designed for morlet wavelet.');

196 end

197

198 if strcmpi(Args.AR1,'auto')

199 for $\mathrm{i}=1: \mathrm{col}$

$200 \quad \operatorname{arc}(\mathrm{i})=\operatorname{ar} \operatorname{lnv}(\mathrm{X}(:, \mathrm{i}))$;

201

end

Args.AR1 $=\operatorname{arc}$

203

if any(isnan(Args.AR1))

204 error('Automatic AR1 estimation failed. Specify it manually (use arcov or arburg).');

205 end

206 end

207

208

$\%$

ANALYZE

209

210

\%Calculate and smooth wavelet spectrum $\mathrm{Y}$ and $\mathrm{X}$

211

212

213

[Y,period,scale,coiy] = wavelet(y(:,2),dt,Args.Pad,Args.Dj,Args.S0,Args.J1,Args.Mother);

$214 \operatorname{sinv}=1 . /\left(\right.$ scale'$\left.^{\prime}\right)$;

215

$\operatorname{smY}=\operatorname{smoothwavelet}\left(\operatorname{sinv}(:, \operatorname{ones}(1, \mathrm{n})) .^{*}\left(\operatorname{abs}(\mathrm{Y}) .^{\wedge} 2\right), \mathrm{dt}\right.$, period,Args.Dj,scale $)$;

dte $=\mathrm{dt}^{*} .01$;

$219 \operatorname{idx}=$ find $((\mathrm{y}(:, 1)>=(\mathrm{t}(1)-\mathrm{dte})) \&(\mathrm{y}(:, 1)<=(\mathrm{t}(\mathrm{end})+\mathrm{dte})))$;

$220 \quad \mathrm{Y}=\mathrm{Y}(:, \mathrm{idx})$;

221

222

smY $=s m Y(:, i d x)$;

223

224

225

226

coiy=coiy $(\mathrm{idx})$;

227

for $\mathrm{i}=2$ :col

228

229

[XS, period,scale, coix] = wavelet(x2(:,(i-1)), dt,Args.Pad,Args.Dj,Args.S0,Args.J1,Args.Mother);

230

$\operatorname{idx}=$ find $((x 1(:,(i-1))>=(t(1))-d t e) \&(x 1(:,(i-1))<=(t(e n d)+d t e)))$;

$\mathrm{XS}=\mathrm{XS}(:, \mathrm{idx})$; 
$\operatorname{coix}=\operatorname{coix}(\mathrm{idx})$;

232

233

XS1(:,;,(i-1))=XS;

$234 \operatorname{coi}=\min ($ coi,coix);

235

236

end

237

238

\% -------- Calculate Cross Wavelet Spectra----------------------------

239

240

241

242

$\%$---- between dependent variable and independent variables------

243

for $\mathrm{i}=1$ :(col-1)

244

$\mathrm{Wyx}=\mathrm{Y} . * \operatorname{conj}(\mathrm{XS} 1(:, ;, \mathrm{i}))$;

245

sWyx $=$ smoothwavelet $(\operatorname{sinv}(:$, ones $(1, \mathrm{n}))$ *Wyx,dt,period,Args.Dj,scale);

246

$\operatorname{sWyx} 1(:,:, i)=s W y x$;

247

248

end

249

$\%$----between independent variables and independent variables------

250

for $i=1:(\operatorname{col}-1)$;

$251 \quad \mathrm{Wxx}_{\mathrm{xx}} \mathrm{XS1} 1(:,, \mathrm{i}) \cdot{ }^{*} \operatorname{conj}(\mathrm{XS} 1(:,, \mathrm{j}))$;

for $\mathrm{j}=1$ :(col-1);

$252 \mathrm{sWxx}=\operatorname{smoothwavelet}\left(\operatorname{sinv}(:, \operatorname{ones}(1, \mathrm{n})) .{ }^{*} \mathrm{Wxx}, \mathrm{dt}\right.$, period,Args.Dj,scale $)$;

$253 \quad s W x x 1(:,:, i, j)=s W x x$;

254 end

255 end

256

257

$\%$ Mutiple wavelet coherence

$258 \%$ calculate the multiple wavelet coherence

259 for $\mathrm{i}=1$ :length(scale)

260 parfor $\mathrm{j}=1: \mathrm{n}$

$261 \mathrm{a}=\operatorname{transpose}(\operatorname{squeeze}(\operatorname{sWyx} 1(\mathrm{i}, \mathrm{j},:)))$;

$262 b=\operatorname{inv}(\operatorname{squeeze}(\operatorname{sWxx} 1(\mathrm{i}, \mathrm{j},:,:)))$;

$263 \mathrm{c}=\operatorname{conj}(\operatorname{squeeze}(\mathrm{sWyx} 1(\mathrm{i}, \mathrm{j},:)))$;

$264 \mathrm{~d}=\operatorname{smY}(\mathrm{i}, \mathrm{j})$;

$265 \operatorname{Rsq}(\mathrm{i}, \mathrm{j})=\operatorname{real}(\mathrm{a} * \mathrm{~b} * \mathrm{c} / \mathrm{d})$;

266 end

267 end

268

269

$\%$------------- make figure-

270

if (nargout>0) $\|($ Args.MakeFigure) 
280 Yticks $=2 .^{\wedge}($ fix $(\log 2(\min ($ period $))):$ fix $(\log 2(\max ($ period $))))$;

if Args.BlackandWhite

colorbarf(cout,H)

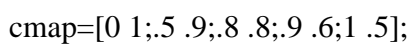

cmap=interp1(cmap $\left.(:, 1), \operatorname{cmap}(:, 2),(0: .1: 1)^{\prime}\right)$;

cmap=cmap $\left(:,\left[\begin{array}{lll}1 & 1 & 1\end{array}\right]\right)$;

298

hold on

if $\sim \operatorname{all}($ isnan (mwcsig))

end

$\%$ suptitle([sTitle ' coherence']);

$\%$ plot(t,log2(coi),'k','linewidth',2)

$$
\mathrm{tt}=\left[\mathrm{t}\left(\left[\begin{array}{ll}
1 & 1
\end{array}\right]\right)-\mathrm{dt} * .5 ; \mathrm{t} ; \mathrm{t}([\text { end end }])+\mathrm{dt}^{*} .5\right] ;
$$

$\%$ hcoi=fill(tt,log2([period([end 1]) coi period([1 end] $)])$ );

\%hatching- modified by $\mathrm{Ng}$ and Kwok

hcoi=fill(tt,log2([period([end 1]) coi period([1 end])]),'w');

hatch(hcoi,45,[0 00$])$;

hatch(hcoi,135,[0 00$]$ );

set(hcoi,'alphadatamapping','direct','facealpha',.5); 


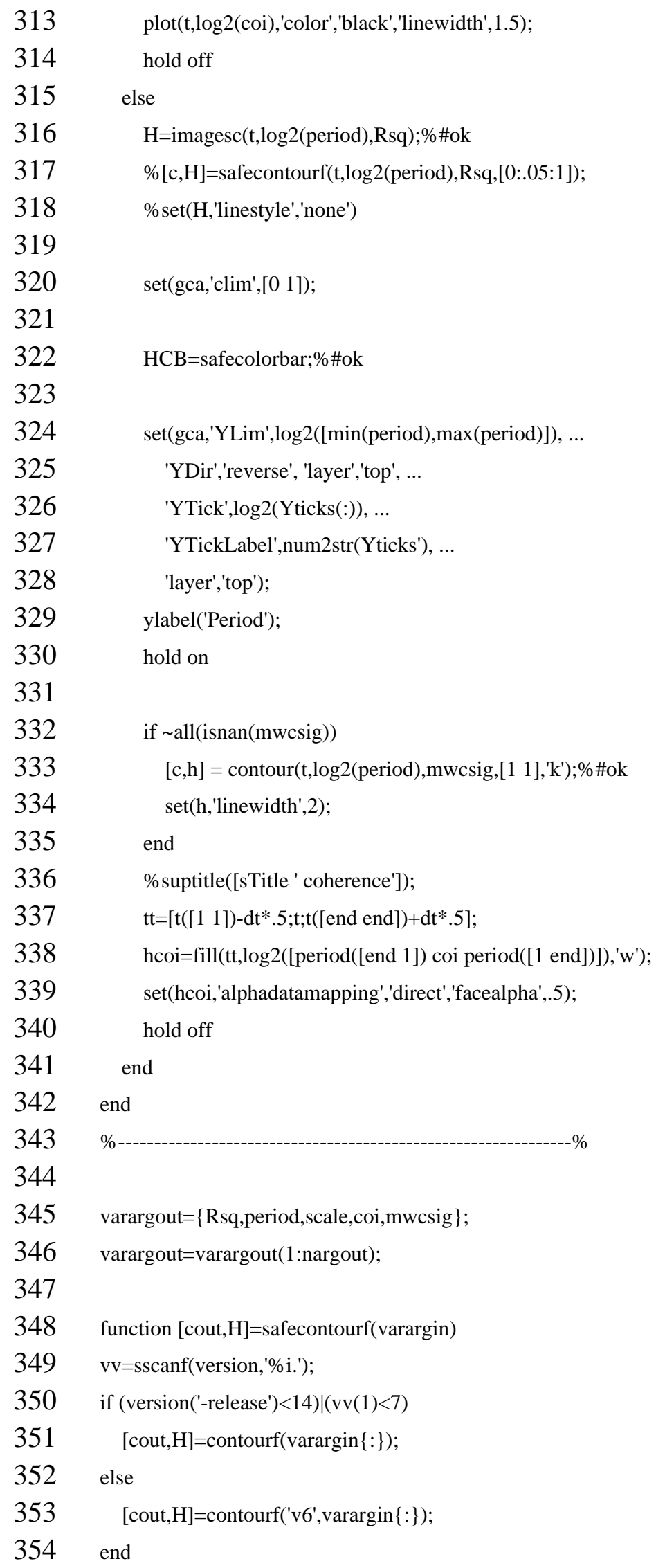

$336 \%$ suptitle([sTitle ' coherence']);

$337 \quad \mathrm{tt}=\left[\mathrm{t}\left(\left[\begin{array}{ll}1 & 1\end{array}\right]\right)-\mathrm{dt} * 5 ; \mathrm{t} ; \mathrm{t}([\mathrm{end}\right.$ end $\left.])+\mathrm{dt} * .5\right]$;

338 hcoi=fill(tt, $\log 2([$ period([end 1]) coi period([1 end])]),'w');

339 set(hcoi,'alphadatamapping','direct','facealpha',.5);

340 hold off

341 end

342 end

345 varargout $=\{$ Rsq,period,scale,coi,mwcsig $\}$;

346 varargout=varargout $(1:$ nargout $)$;

348 function [cout,H]=safecontourf(varargin)

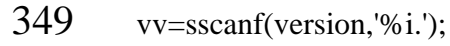

350 if (version('-release')<14)|(vv(1)<7)

$351 \quad[$ cout, $\mathrm{H}]=$ contourf $(\operatorname{varargin}\{:\})$;

352 else

$353 \quad[$ cout, $\mathrm{H}]=$ contourf('v6', varargin $\{:\})$;

354 end 
function $\mathrm{hcb}=$ safecolorbar(varargin)

357

$\mathrm{vv}=$ sscanf(version,'\%i.');

358

359 if (version('-release') $<14) \mid(\operatorname{vv}(1)<7)$

$360 \mathrm{hcb}=\mathrm{colorbar}(\operatorname{varargin}\{:\})$;

361 else

362 hcb=colorbar('v6', varargin $\{:\})$;

363 end 


\section{S3 Matlab code for significance test on multiple wavelet coherence}

$365 \%$ This is a Matlab file (mwcsignif.m) for calculating significance tests on multiple wavelet coherence.

366 \%Please copy the following content into a txt file and rename this file to "mwcsignif.m" prior to running.

368 function mwcsig=mwcsignif(mccount,ar1,dt,n,pad,dj,s0,j1,mother,cutoff)

$369 \%$ Multiple Wavelet Coherence Significance Calculation (Monte Carlo)

$370 \%$

$371 \%$ mwcsig=mwcsignif(mccount,ar1,dt,n,pad,dj,s0,j1,mother,cutoff)

$372 \%$

$373 \%$ mccount: number of time series generations in the monte carlo run

$374 \%$ (the greater the better)

$375 \%$ ar1: a vector containing two (in case of calculating wavelet

$376 \%$ coherence between two variables) or

$377 \%$ multiple $(\geq 3)$ (in case of calculating multiple wavelet coherence

$378 \%$ with three or more variables)

$379 \%$ AR1 coefficients.

$380 \%$ dt,pad,dj,s0,j1, mother: see wavelet help...

$381 \%$ n: length of each generated timeseries. (obsolete)

$382 \%$

$383 \%$ cutoff: (obsolete)

$384 \%$

$385 \%$ RETURNED

$386 \%$ mwcsig: the $95 \%$ significance level as a function of scale... (scale,sig95level)

$387 \%$

$388 \%$ Please acknowledge the use of this software package in any publications,

$389 \%$ by including text such as:

$390 \%$

$391 \%$ "The software for the multiple wavelet coherence was provided by W. Hu

$392 \%$ and B. Si, and is available in the supplement of $\mathrm{Hu}$ and $\mathrm{Si} \mathrm{(2016)."}$

$393 \%$ and cite the paper:

$394 \%$ "Hu, W., and B. Si (2016), Technical Note: Multiple wavelet coherence for untangling scale-specific and localized

$395 \%$ multivariate relationships in geosciences, Hydrol. Earth Syst. Sci., volume and page numbers to be allocated ."

$396 \%$

$397 \%$ (C) W. Hu and B. C. Si 2016

$398 \%$

$399 \%$

$400 \%$

$401 \%$ Copyright (C) 2016, W. Hu and B. C. Si 2016

$402 \%$ This software may be used, copied, or redistributed as long as it is not

$403 \%$ sold and this copyright notice is reproduced on each copy made. This

$404 \%$ routine is provided as is without any express or implied warranties 
try

438 if (last.mccount $>=$ mccount) \&\& (isequal(checkvalues,last.checkvalues))

439 mwcsig=last.mwcsig;

$440 \quad$ return

$441 \quad$ end

442 catch

443 end

$445 \%$ choose a n so that largest scale have atleast some part outside the coi

$446 \mathrm{~ms}=\mathrm{s} 0 *\left(2^{\wedge}(\mathrm{j} 1 * \mathrm{dj})\right) / \mathrm{dt} ; \%$ maxscale in units of samples 
$\mathrm{n}=\operatorname{ceil}\left(\mathrm{ms}^{*} 6\right)$;

448

449

warned $=0$;

$450 \%$ precalculate stuff that's constant outside the loop

$451 \% \mathrm{~d} 1=\operatorname{ar} 1 \mathrm{noise}(\mathrm{n}, 1, \operatorname{ar} 1(1), 1)$;

$452 \mathrm{~d} 1=$ rednoise(n, ar1(1),1);

453 [W1,period,scale,coi] $=$ wavelet $(\mathrm{d} 1, \mathrm{dt}, \mathrm{pad}, \mathrm{dj}, \mathrm{s} 0, \mathrm{j} 1$, mother $)$;

454 outsidecoi=zeros( $\operatorname{size}(\mathrm{W} 1))$;

455 for $\mathrm{s}=1$ :length(scale)

456 outsidecoi $(\mathrm{s},:)=(\operatorname{period}(\mathrm{s})<=\mathrm{coi})$;

457 end

$458 \quad \operatorname{sinv}=1 . /\left(\right.$ scale'$\left.^{\prime}\right)$;

$459 \operatorname{sinv}=\operatorname{sinv}(:$, ones $(1, \operatorname{size}(\mathrm{W} 1,2)))$;

460

461

if mccount $<1$

462 mwcsig=scale';

$463 \operatorname{mwcsig}(:, 2)=.71 ; \%$ pretty $\operatorname{good}$

464 return

465 end

466

467

sig95=zeros $($ size $($ scale $))$;

468

469

maxscale $=1$;

470

for $\mathrm{s}=1$ :length(scale)

471

if any(outsidecoi(s,:)>0)

472

maxscale=s;

473

else

474

475

$\operatorname{sig} 95(\mathrm{~s})=\mathrm{NaN}$;

476

if $\sim$ warned

477

warning('Long wavelengths completely influenced by COI. (suggestion: set $\mathrm{n}$ higher, or j1 lower)');

478 warned $=1$;

479

480 end

481

482

483

\%PAR1=1./ar1spectrum(ar1(1),period');

$484 \%$ PAR2=1./ar1spectrum $(\operatorname{ar} 1(2)$,period' $)$

$485 \%$ \%AR2=PAR2(:,ones $(1, \operatorname{size}(\mathrm{W} 1,2)))$;

486

487

nbins $=1000$;

$488 \quad$ wlc=zeros(length(scale),nbins); 
513 Wdyx=Wdy. *conj(Wdx1(:,:,i));

514 sWdyx=smoothwavelet $(\operatorname{sinv}(:$,ones $(1, \mathrm{n}))$. $*$ Wdyx,dt,period, dj,scale);

515 sWdyx $1(:,:, \mathrm{i})=\mathrm{sWdyx}$;

516 end

517

$518 \%$----between independent variables and independent variables------

519 for $\mathrm{i}=1:(\mathrm{col}-1)$;

520 parfor $\mathrm{j}=1:(\mathrm{col}-1)$;

$521 \quad \mathrm{Wdxx}=\mathrm{Wdx} 1(:, ;, \mathrm{i}) . * \operatorname{conj}(\operatorname{Wdx} 1(:, ;, \mathrm{j}))$;

$522 \operatorname{sWdxx}=\operatorname{smoothwavelet}(\operatorname{sinv}(:$, ones $(1, \mathrm{n})) . * \mathrm{Wdxx}, \mathrm{dt}$, period,dj,scale $)$;

523 sWdxx1(:,:,i,j)=sWdxx;

524 end

525 end

526

$527 \%$ calculate the multiple wavelet coherence

528 for $\mathrm{i}=1$ :length(scale)

529 parfor $\mathrm{j}=1: \mathrm{n}$

530 a=transpose(squeeze(sWdyx1(i,j,:))); 
$\mathrm{b}=\operatorname{inv}(\operatorname{squeeze}(\operatorname{sWdxx} 1(\mathrm{i}, \mathrm{j},:,:)))$;

532

$\mathrm{c}=\operatorname{conj}(\operatorname{squeeze}(\operatorname{sWdyx} 1(\mathrm{i}, \mathrm{j},:)))$;

533

$\mathrm{d}=\operatorname{smd} \mathrm{Y}(\mathrm{i}, \mathrm{j})$;

$534 \quad \operatorname{Rsq}(\mathrm{i}, \mathrm{j})=\operatorname{real}(\mathrm{a} * \mathrm{~b} * \mathrm{c} / \mathrm{d})$;

535 end

536 end

537

538

539

for $\mathrm{s}=1$ :maxscale

540

$\mathrm{cd}=\operatorname{Rsq}(\mathrm{s}$, find $($ outsidecoi(s,:)));

541

$\mathrm{cd}=\max (\min (\mathrm{cd}, 1), 0)$;

542

$\mathrm{cd}=$ floor $\left(\mathrm{cd}^{*}(\right.$ nbins -1$\left.)\right)+1$;

543

for $\mathrm{jj}=1$ :length $(\mathrm{cd})$

544 end

545 end

546 end

547 close(wbh);

548

549 $w l c(s, c d(j j))=w l c(s, c d(j j))+1 ;$

$550 \quad$ rsqy=((1:nbins $)-.5) /$ nbins;

551 ptile=wlc(s,:);

$552 \mathrm{idx}=$ find $($ ptile $\sim=0$ );

553 ptile=ptile (idx);

$554 \quad$ rsqy=rsqy $(\mathrm{idx})$;

555 ptile=cumsum(ptile);

556 ptile= $=($ ptile-.5)/ptile(end);

$557 \quad$ sig95(s)=interp1(ptile,rsqy,.95);

558 end

559 mwcsig=[scale' sig95'];

560

561 if any(isnan(sig95))\&( warned)

562 warning('Sig95 calculation failed. (Some NaNs)');

563 else

564 try

565 save(cachefilename,'mccount','checkvalues','mwcsig'); \%save to a cache....

566 catch

567 warning(['Unable to write to cache file: ' cachefilename]);

568 end

569 end 
572 Multiple wavelet coherence package

573 by Wei Hu and Bingcheng $\mathrm{Si}$

574 Release date: 27 April 2016

576 This software package is written for performing multiple wavelet coherence.

577 This software package includes mwc.m and mwcsignif.m, which

578 are written in the Matlab program based on wtc.m and wtcsignif.m provided by A.

579 Grinsted

580 (http://www.glaciology.net/wavelet-coherence).

582 Users are, therefore, required to download his software package and

583 combine these two packages into one to run the multiple wavelet coherence analysis. In 584 the package provided by A. Grinsted, Matlab codes included are anglemean.m, arl.m, 585 arlnv.m, boxpdf.m, formatts.m, normalizepdf.m, phaseplot.m, smoothwavelet.m, wt.m, 586 wtc.m, wtcdemo.m, wtcsignif.m, xwt.m.

588 Please acknowledge the use of this software package in any publications by including 589 text such as: The software for the multiple wavelet coherence was provided by W. Hu and B. C. Si, and is available in the supplement of $\mathrm{Hu}$ and $\mathrm{Si} \mathrm{(2016).}$

594 and cite the paper:

$595 \% \% \% \% \% \% \% \% \% \% \% \% \% \% \% \% \% \% \% \% \% \% \% \% \% \% \% \% \% \% \% \% \% \% \% \% \% \% \%$

596 Hu, W., and B.C. Si (2016), Technical Note: Multiple wavelet coherence for untangling 597 scale-specific and localized multivariate relationships in geosciences, Hydrol. Earth Syst.

598 Sci., volume and page numbers to be allocated.

599 \%\%\%\%\%\%\%\%\%\%\%\%\%\%\%\%\%\%\%\%\%\%\%\%\%\%\%\%\%\%\%\%\%\%\%\%\%\%\%\%\%\% 
602 Acknowledgements:

603

604 Wavelet software was provided by C. Torrence and G. Compo,

605 and is available at URL: http://paos.colorado.edu/research/wavelets/.

606

607 Crosswavelet and wavelet coherence software were provided by

608 A. Grinsted and is available at URL:

609 http://www.glaciology.net/wavelet-coherence

610

611 Should there be any enquiries, please feel free to contact:

612

613 Wei Hu

614 Email: wei.hu@plantandfood.co.nz

615

616 Bing Si

617 Email: bing.si@usask.ca 


\section{S5 Results of MEMD}

619 Six or seven intrinsic mode functions (IMFs) corresponding to different scales are

620 obtained for multivariate data series (i.e., a combination of the response variable with two

$621\left(y_{2}\right.$ and $y_{4}$, or $z_{2}$ and $\left.z_{4}\right)$ or three $\left(y_{2}, y_{3}\right.$, and $y_{4}$, or $z_{2}, z_{3}$, and $\left.z_{4}\right)$ predictor variables) by

622 MEMD. Due to IMFs, with a number of 6 or greater, contributing negligible variance to

623 the total, only the first five IMFs are presented (Fig. S1). For each IMF, the scale is

624 calculated as the total number of points (i.e., 256) divided by the number of cycles for

625 each IMF. The obtained scales and percentage (\%) of variance explained by each IMF are

626 shown in Table S1. While the obtained scales for the response variable $y$ are in agreement

627 with the true scales for the stationary case, the obtained scales (i.e., 3, 6, 11, 21, and 43)

628 for the response variable $z$ deviate slightly from the average scales for the non-stationary

629 case. For the response variable, the contribution of IMFs to the total variance generally

630 decreases (20\% to $13 \%$ for stationary and $27 \%$ to $11 \%$ for non-stationary) from IMF1 to

631 IMF5, which disagrees with the fact that each scale contributes equally (i.e., 20\%) to the

632 total variance. The scale of the dominant variance from each predictor variable can be

633 obtained (Table S1). However, the sum of variances over all IMFs for each variable is

634 less than $100 \%$ (ranging from $84 \%$ to $93 \%$ ), indicating that MEMD cannot capture all the

635 variances, as was also previously observed (Hu et al., 2013; She et al., 2014). 

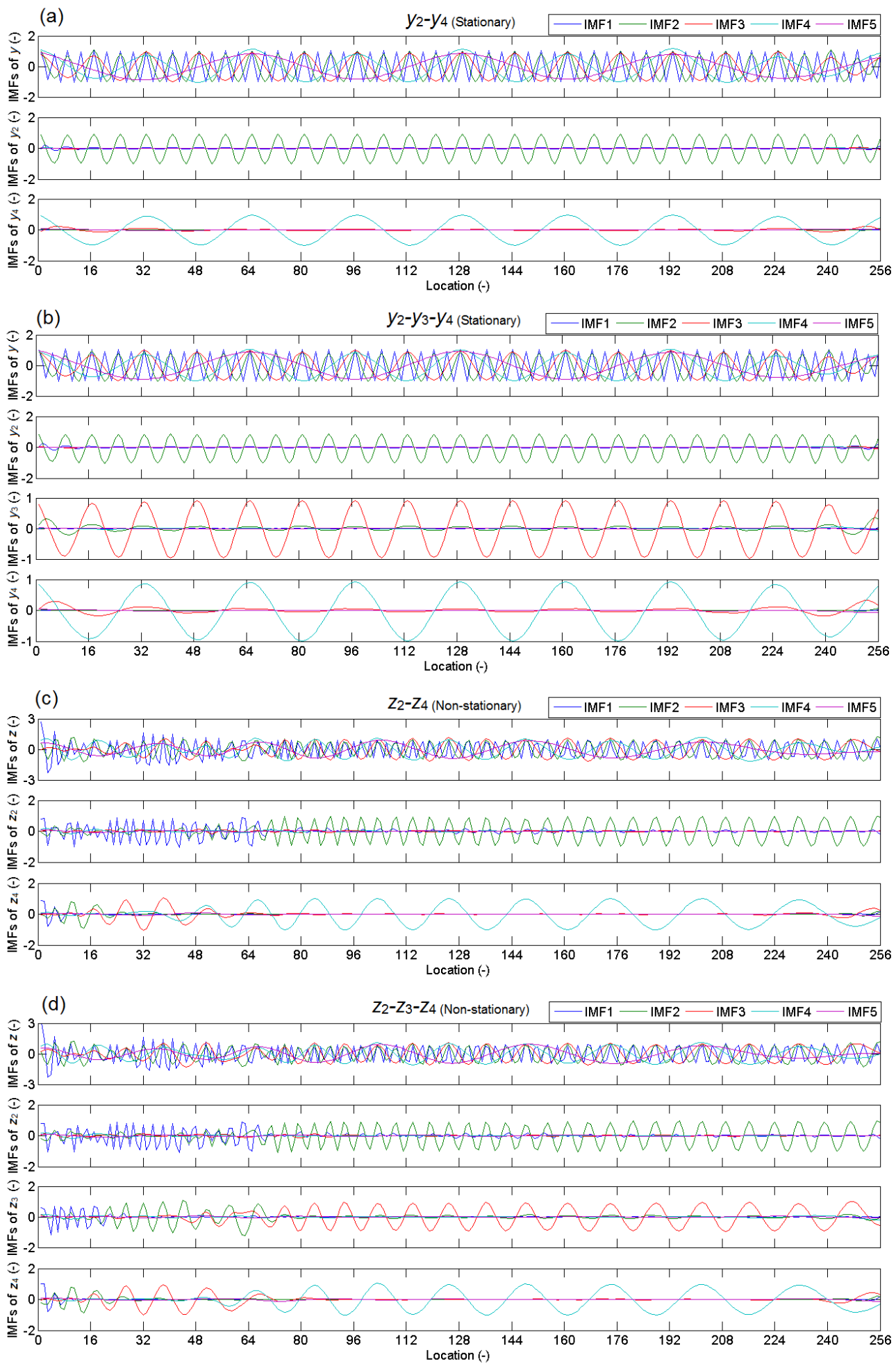

Figure S1. The first five intrinsic mode functions (IMFs) of response variable $y$ (or $z$ ) and predictor variables $\left(y_{2}\right.$ and $y_{4} ; y_{2}, y_{3}$, and $y_{4} ; z_{2}$ and $z_{4} ;$ or $z_{2}, z_{3}$, and $\left.z_{4}\right)$ obtained by multivariate empirical mode decomposition. 
643 Table S1. Scales and percentage (\%) of variance explained by each intrinsic mode 644 function (IMF) of response variable $y$ (or $z$ ) and predictor variables $\left(y_{2}\right.$ and $y_{4} ; y_{2}, y_{3}$ and $645 y_{4} ; z_{2}$ and $z_{4} ;$ or $z_{2}, z_{3}$, and $\left.z_{4}\right)$ using the multivariate empirical mode decomposition 646 method.

647

\begin{tabular}{|c|c|c|c|c|c|c|}
\hline & & Scale (-) & $y(\%)$ & $y_{2}(\%)$ & $y_{3}(\%)$ & $y_{4}(\%)$ \\
\hline \multirow[t]{5}{*}{$y_{2}-y_{4}($ Stationary $)$} & IMF1 & 4 & 20 & 0 & & 0 \\
\hline & IMF2 & 8 & 18 & 90 & & 0 \\
\hline & IMF3 & 16 & 15 & 0 & & 1 \\
\hline & IMF4 & 32 & 18 & 0 & & 88 \\
\hline & IMF5 & 64 & 13 & 0 & & 0 \\
\hline \multirow[t]{5}{*}{$y_{2}-y_{3}-y_{4}($ Stationary $)$} & IMF1 & 4 & 20 & 1 & 0 & 0 \\
\hline & IMF2 & 8 & 17 & 85 & 1 & 0 \\
\hline & IMF3 & 16 & 16 & 0 & 82 & 2 \\
\hline & IMF4 & 32 & 16 & 0 & 0 & 82 \\
\hline & IMF5 & 64 & 15 & 0 & 0 & 0 \\
\hline \multirow[t]{5}{*}{$z_{2}-z_{4}$ (Non-stationary) } & IMF1 & 3 & 27 & 22 & & 2 \\
\hline & IMF2 & 6 & 17 & 68 & & 4 \\
\hline & IMF3 & 11 & 17 & 0 & & 11 \\
\hline & IMF4 & 21 & 17 & 0 & & 75 \\
\hline & IMF5 & 43 & 11 & 0 & & 0 \\
\hline \multirow[t]{5}{*}{$z_{2}-z_{3}-z_{4}$ (Non-stationary) } & IMF1 & 3 & 27 & 22 & 7 & 3 \\
\hline & IMF2 & 6 & 18 & 69 & 17 & 4 \\
\hline & IMF3 & 11 & 17 & 0 & 61 & 14 \\
\hline & IMF4 & 21 & 16 & 0 & 1 & 68 \\
\hline & IMF5 & 43 & 11 & 0 & 0 & 0 \\
\hline
\end{tabular}

648

649

650

651 
652

653

654

655

656

657

658

659

660 around 1 year, relative humidity was positively correlated to $E$ during the period of 1979

661 to 1997 . This is due to high relative humidity usually being associated with high summer

662 temperatures, when high evaporation occurs. At other scales (e.g., 2-6 months or 5-10

663 years), the relative humidity was negatively correlated to the $E$, which was expected. The

664 dominant factors explaining variation in $E$ differed with scale. For example, the relative

665 humidity was the dominating factor at small (2-8 months) and large (>32 months) scales,

666 while temperature was the dominating factor at the medium (8-32 months) scales (Fig.

667 S2). The relative humidity corresponded to the greatest mean MWC (0.62) and PASC

668 value $(40 \%)$ at multiple scale-location domains. 
(a) Relative humidity

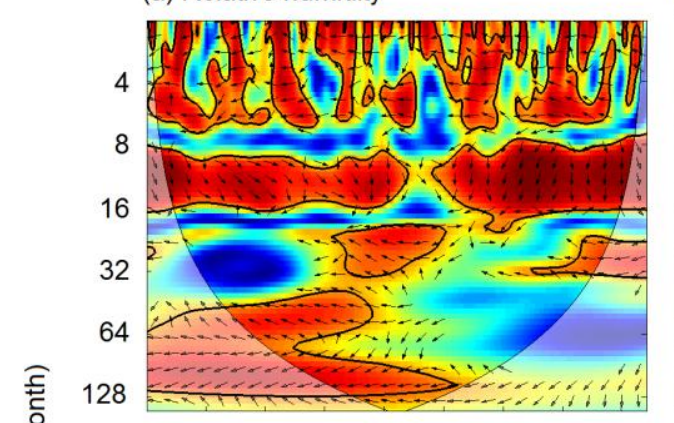

(c) Sun hours

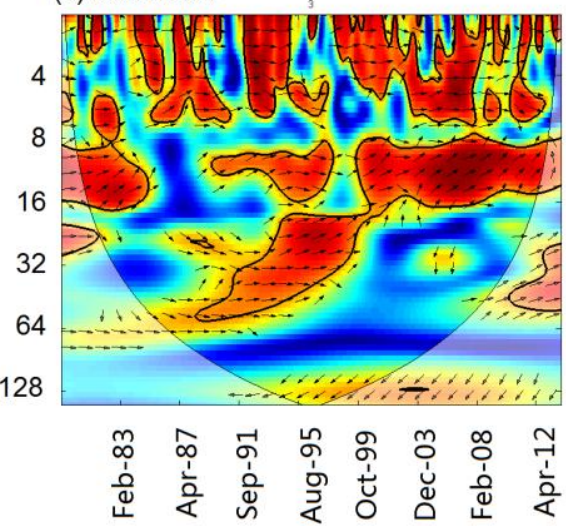

(b) Mean temperature

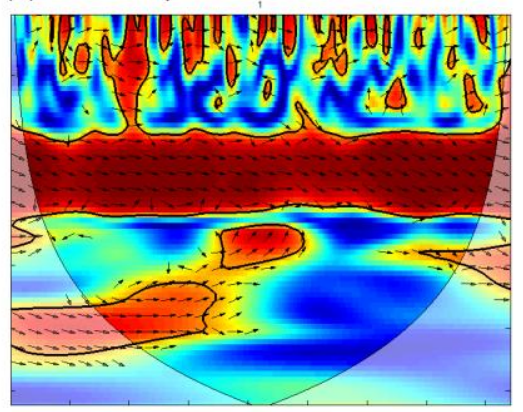

(d) Wind speed
670

671

672

673

674

675

676

677

678

679

680

681

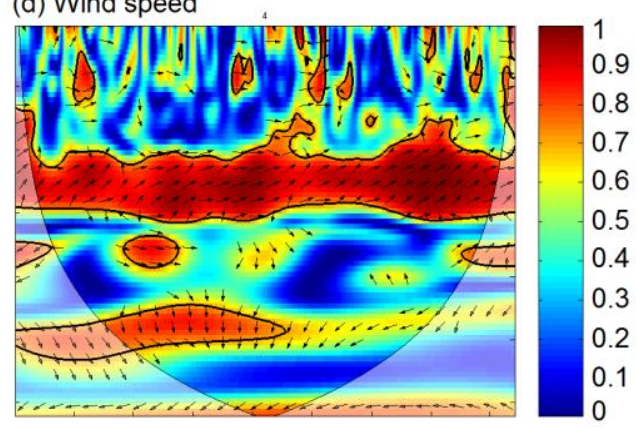

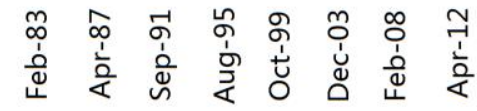

Time (month-year)

Figure S2. Bivariate wavelet coherency between evaporation $(E)$ from water surfaces and each of the meteorological factors (relative humidity, mean temperature, sun hours, and wind speed) at Changwu site in Shaanxi, China. Arrows show the correlation type with the right pointing arrows being positive and left pointing arrows being negative. Thin solid lines demarcate the cones of influence and thick solid lines show the 95\% confidence levels. 
684 Das, N.N. and Mohanty, B. P.: Temporal dynamics of PSR-based soil moisture across 685 spatial scales in an agricultural landscape during SMEX02: A wavelet approach, Remote 686 Sens. Environ., 112, 522-534, doi:10.1016/j.rse.2007.05.007, 2008.

687 Grinsted, A., Moore, J. C., and Jevrejeva, S.: Application of the cross wavelet transform 688 and wavelet coherence to geophysical time series, Nonlinear Proc. Geoph., 11, 561-566, 6892004.

$690 \mathrm{Hu}, \mathrm{W}$. and Si, B. C.: Soil water prediction based on its scale-specific control using 691 multivariate empirical mode decomposition, Geoderma, 193-194,180-188, doi:

692 10.1016/j.geoderma.2012.10.021, 2013.

693 Kumar, P. and Foufoula-Georgiou, E.: Wavelet analysis for geophysical applications, 694 Rev. Geophys., 35, 385-412, doi: 10.1029/97RG00427, 1997.

695 She, D. L., Tang, S. Q., Shao, M. A., Yu, S. E., and Xia, Y. Q.: Characterizing scale 696 specific depth persistence of soil water content along two landscape transects, J. Hydrol., 697 519, 1149-1161, doi:10.1016/j.jhydrol.2014.08.034, 2014.

698 Si, B. C.: Spatial scaling analyses of soil physical properties: A review of spectral and 699 wavelet methods, Vadose Zone J., 7, 547-562, doi: 10.2136/vzj2007.0040, 2008.

700 Torrence, C. and Compo, G. P.: A practical guide to wavelet analysis, Bull. Am.

701 Meteorol. Soc., 79, 61-78, doi: 10.1175/1520-0477(1998)079<0061:apgtwa>2.0.co;2, 7021998. 
703 Torrence, C. and Webster, P. J.: Interdecadal changes in the ENSO-monsoon system, J.

704 Clim., 12, 2679-2690, doi: 10.1175/1520-0442(1999)012<2679:ICITEM>2.0.CO;2,

7051999. 